\title{
Prensado, con métodos de la cerámica plana, de piezas cerámicas curvas con especificaciones geométricas de elevada precisión
}

\author{
J. SERRANO ${ }^{1}$, J. M. CASTELLET ${ }^{1}$, R. BONAQUE ${ }^{2}$, A. POYATOS ${ }^{2}$, F. ROMERO ${ }^{1}$ \\ ${ }^{1}$ Universitat Jaume I, Castellón, España \\ ${ }^{2}$ Macer S.L., Almazora (Castellón), España
}

\begin{abstract}
Este trabajo ha sido presentado como comunicación oral, tras su evaluación por el Comité Científico, en el XII Foro Global del Recubrimiento Cerámico. QUALICER (13 y 14 febrero 2012. Castellón. España).
\end{abstract}

\begin{abstract}
Para obtener piezas tridimensionales de elevada precisión por prensado en seco se plantea dos líneas: modelar el flujo del material y la deformación durante el prensado; evaluar la distribución de Da en una pieza prensada y usar modelos de contracción que permitan predecir la geometría tras la cocción. Así se podría intervenir en la fase de diseño (geometría funcional y reverso) y del proceso.

Este trabajo expone los estudios llevados a cabo para predecir la geometría final de piezas tridimensionales de tipo lámina obtenidas por técnicas convencionales de prensado, a partir de la distribución de Da en cada punto de la pieza. Para ello se modelaron piezas por el método de los elementos finitos (MEF, en inglés FEM), aplicándoseles procesos de simulación para obtener la deformación en cada punto de la pieza en función de su Da. La validación experimental se realizó usando el método de absorción de RX para la Da y una MMC (máquina de medición por coordenadas) para la geometría.
\end{abstract}

Palabras clave: Prensado piezas curvas, compensación deformaciones anisótropas, cocción piezas complejas, control densidad aparente $(D a)$

Pressing, with flat ceramics methods, of curved ceramic parts with high-precision geometry specifications

In order to obtain high-precision three-dimensional pieces by dry pressing, two approaches were studied: modelling the material flow and strain during pressing; and evaluating the bulk density (Da) distribution in a pressed piece and using shrinkage models that allow the post-firing geometry to be predicted. It would thus become possible to act in the design phase (functional geometry and rear) and in the process.

This paper describes the studies carried out to predict the final geometry of three-dimensional pieces of the sheet type obtained by conventional pressing techniques, from the Da distribution in each point of the piece. For this purpose, pieces were modelled using finite element method (FEM), applying simulation processes to obtain the strain at each point of the piece as a function of its Da. The experimental validation was performed using the X-ray absorption method for Da and a CMM (coordinate measuring machine) for the geometry.

Keywords: Curved ceramics pressing, anisotropic deformations compensation, complex tile firing, bulk density control (Da)

\section{INTRODUCCIÓN}

Durante la cocción de una baldosa prensada en seco se produce una contracción respecto a su geometría en verde, que principalmente depende del material y de su densidad aparente (Da). Sabido es que a cada material le corresponde un diagrama de gresificación que da valores de contracción en función de la $\mathrm{Da}$, y que variaciones de densidad aparente $(\Delta \mathrm{Da})$ entre piezas producen dimensiones diferentes, y que estas variaciones en diferentes zonas de una misma pieza origina errores geométricos.

Para reducir la $\Delta \mathrm{Da}$, y con ello la variabilidad mejorando la precisión, se recurre al empleo de punzones isostáticos y al control del llenado de los alvéolos. Pero estas técnicas, pensadas para piezas cuasi planas, resultan de difícil aplicación en piezas tridimensionales (diferencias de altura en relieve $\geq 15 \mathrm{~mm}$ ); más aún si se requiere elevada precisión dimensional.
Además, la expansión post-prensado y la contracción postcocción se manifiestan de forma no lineal en las láminas curvas, precisándose modelos que permitan estimar cuál debería ser la geometría en verde para una geometría final cocida dada. El modelo lineal usado para lámina plana (un simple zoom) no es aplicable para obtener geometrías tridimensionales precisas.

Han de adaptarse los métodos de ejecución de la cerámica plana. Así, si el llenado del alvéolo es plano, la obtención de piezas tridimensionales de espesores cuasi uniformes (láminas) requiere grandes desplazamientos del polvo depositado. Además, los granos de atomizado son huecos y al prensar se deforman plásticamente en lugar de únicamente desplazarse, contra lo que sucedería con gránulos macizos. Así, la obtención de piezas tridimensionales por prensado en seco, usando técnicas actuales, resulta compleja. 


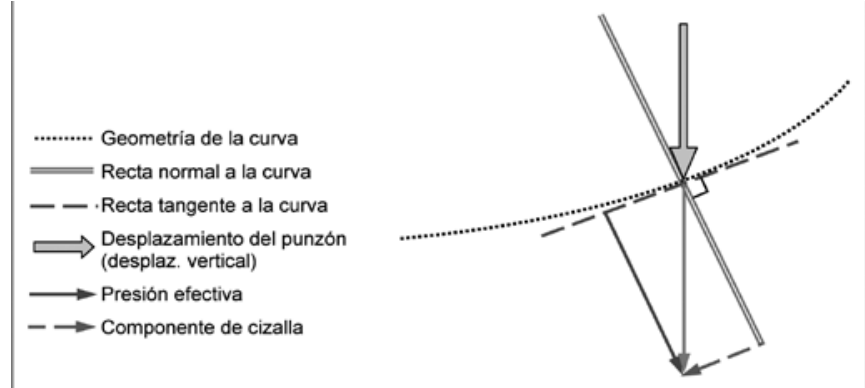

Figura 1. Componentes de la fuerza de prensado en superficies curvas.

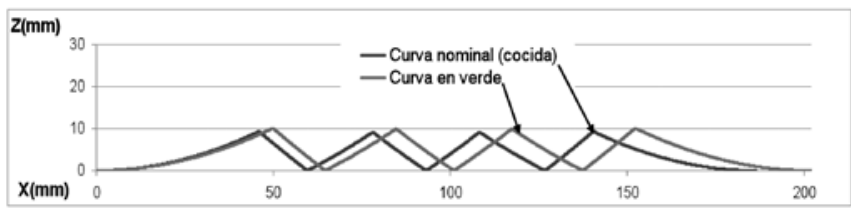

Figura 2. Curva de la cara noble: nominal y verde (no está a escala)

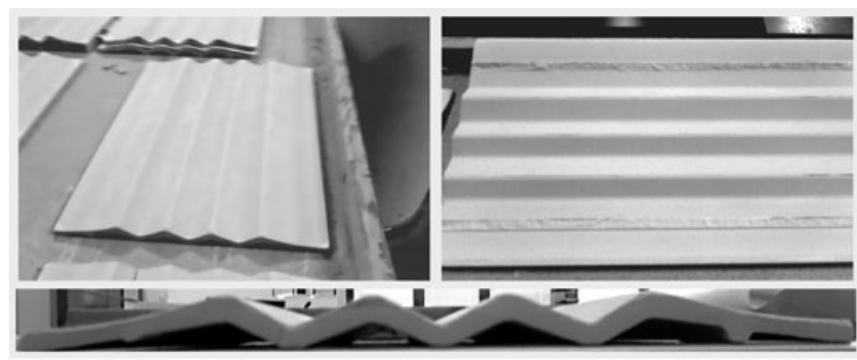

Figura 3. Prototipos prensados de pieza con reverso compensado (reservorio)
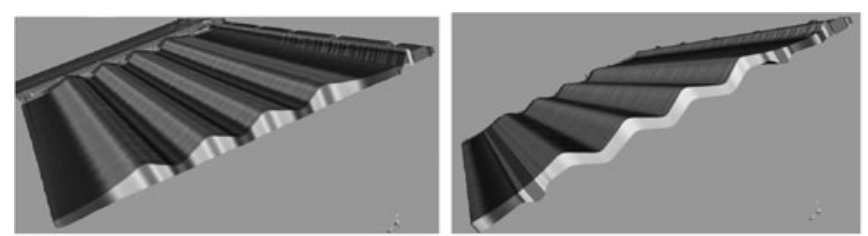

Figura 4. Medida del espesor en verde. Reverso plano y con reservorio

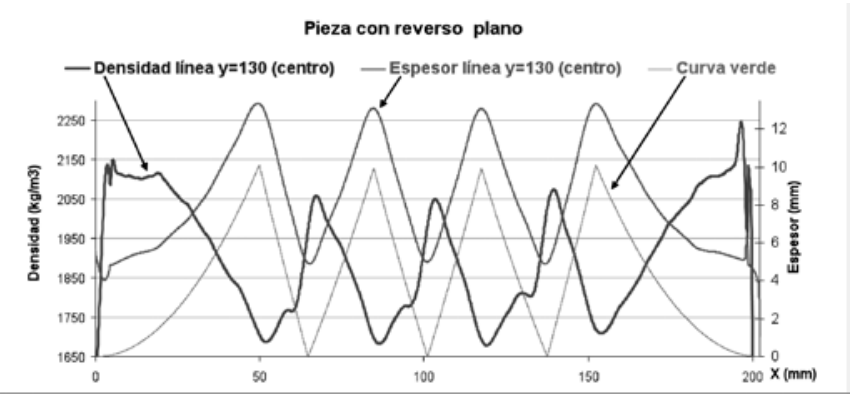

Figura 5. Da y espesor en una pieza con reverso plano

\section{PRENSADO UNIAXIAL CON PUNZONES CURVOS}

En el prensado uniaxial con punzones de superficie curva, una vez el polvo ha entrado en contacto con la superficie, aparece una componente de cizalla que se manifiesta en un movimiento radial del polvo (respecto a la normal a la superficie del punzón) y menor presión efectiva (Pe, componente normal a la superficie de la curva), ambas dependientes de la curvatura y, por tanto, de la posición (véase Figura 1). Ello tiene efecto sobre la compactación y la Da de la pieza en verde.

Para compensarlo, hay que maniobrar con los instrumentos habituales en la industria. Así, conociendo el coeficiente de contracción en cada punto, se puede compensar la curva de la cara noble. No obstante, comoquiera que la geometría del molde introduce restricciones al movimiento esperado del polvo, la compensación no es sencilla.

Otra posibilidad es modificar la geometría del reverso con elementos que limiten el movimiento no deseado de la pasta o habilitando reservorios que acepten la masa desplazada para que ésta no afecte demasiado a la $\Delta \mathrm{Da}$ en el volumen de la pieza. El ajuste de estos procedimientos sigue un laborioso proceso de prueba y error.

En cualquier caso, hay un significativo movimiento radial del polvo (respecto al eje del punzón) que induce gran cantidad de tensión-deformación residual, incrementando, por tanto, las posibilidades de fallo mecánico.

\subsection{Resultados experimentales}

Se han prensado, en una SACMI 980, piezas de gres porcelánico con polvo Arciblansa ESM70, cargando el alvéolo de forma tradicional y enrasando con el marco. El perfil es curvo para la cara noble en el punzón inferior, como en la Figura 2, variando el del reverso (superior y móvil). Durante las primeras pruebas de prensado se observó la necesidad de suavizar el perfil de los nervios de apoyo, o incluso su eliminación, para garantizar la fabricabilidad.

Posteriormente se ha medido por RX, con el Densexplorer ITC (1), la Da (media vertical) en varios cortes transversales para estudiar su dispersión y cómo afectaba la geometría del reverso a su distribución. En (2) se describe el uso de la tomografía axial computarizada de $\mathrm{RX}$ para el estudio de la Da en el volumen de las píldoras farmacéuticas, lo que aporta información sobre la distribución espacial. Aunque las piezas estudiadas se pueden considerar, básicamente, planas, sería de gran utilidad comprobar la distribución de Da en altura para estudiar el efecto de la fricción del polvo con el molde y punzón. En Figura 4, Figura 5 y Figura 6 se pueden observar algunos resultados obtenidos para la medición de la Da con el Densexplorer para dos configuraciones.

De la distribución en el plano de la Da para los especímenes estudiados se ha extraído información relevante para el proceso de prensado:

1. Hay movimiento de la pasta en el alvéolo. Ello se aprecia comparando la variación de la Da en la serie prensada con reverso plano con la realizada con reverso compensado. Las diferencias de Da son de aproximadamente $400 \mathrm{~kg} / \mathrm{m}^{3}$ para las piezas $\mathrm{sin}$ compensación. Con la idea del movimiento de la 
arcilla en mente, se diseñó la compensación del punzón, obteniéndose una mejora en las diferencias que pasaron a unos $200 \mathrm{~kg} / \mathrm{m}^{3}$. Volviendo a modificar la geometría del reverso, se ha llegado a $\Delta \mathrm{Da}$ del orden de los $130 \mathrm{~kg} / \mathrm{m}^{3}$ y las piezas en verde se ajustan bien a la geometría teórica.

2. Se confirmó la observación experimental de que las crestas se prensan menos que los valles (agrietamiento en los valles). Nótese que se prensa con la cara noble abajo, y, en esa situación (cresta en posición inferior):

- Para el caso del prensado con reverso compensado, cabe esperar que el punzón superior, en forma de cuña, desplace la pasta de la cresta (en la parte baja) hacia el valle, donde queda confinada, aumentando la densidad aparente como resultado de esa acumulación.

- Para la reverso plano, el espesor presenta un máximo en la cresta, por lo que la relación de compactación (Rc) es mayor en las crestas que en los valles (a mayor Rc, menor compactación), definiéndose la relación de compactación como: $\mathrm{Rc}=\mathrm{espesor}$ final / (espesor_final+altura_carga).

3. La diferencia de Da parece estar relacionada con la diferencia de espesor. Por ello, parece conveniente igualar espesores, esto es, que la pieza tenga una lámina de espesor medianamente uniforme. Existe una razonable correlación entre el espesor y la densidad aparente (Da); a mayor espesor menos Da. Véase Figura 7 y Figura 8.

\section{Relación Da y espesor}

Analizando los datos experimentales obtenidos por el Densexplorer, se ha determinado la existencia, para una presión y pasta cerámica dada, de una relación lineal entre la Da obtenida y la altura de la carga de pasta cerámica. Ante el riesgo de falta de fiabilidad de los valores de la Da obtenidos en los flancos de subida debido a posibles problemas de alineación de los telémetros del Densexplorer, se han seleccionado únicamente los datos de los flancos de bajada, obteniéndose buenos coeficientes de correlación lineal.

\section{RESULTADOS Y DISCUSIÓN}

Es posible encontrar situaciones parecidas a las descritas para punzones convexos en la industria farmacéutica. Hay que resaltar, sin embargo, algunas diferencias importantes en el compactado estudiado:

a). La relación de aspecto (altura del espécimen dividida por la anchura) de las píldoras es mayor. Volumen vs. lámina (espacio vs. plano).

b). La flecha de las curvas (diferencia de altura entre la cresta y el valle) respecto a la altura total es mayor para las baldosas.

c). La pendiente de las curvas es mayor para las baldosas.
En (3) se indica que las fricciones entre el polvo y el molde y entre el polvo y el punzón tienen un marcado efecto en la distribución espacial de la Da: "Cuando el material en la periferia es inicialmente prensado por la parte exterior del punzón, comienza a densificarse. Una región de alta Da se forma en la esquina superior del compactado, la cual tiende a propagarse vertical y radialmente. Si la fricción es alta, el movimiento radial se restringe y la densificación que sigue se limita a la periferia. La parte central de la tableta comienza a densificarse sólo cuando el punzón superior contacta totalmente el material. Con baja fricción, la tendencia de las regiones inicialmente densificadas a desplazarse radialmente hacia el centro de la tableta es facilitada por el deslizamiento en la cara curvada del molde, llevando a mayores Da en el centro del espécimen. En general, el alcance de este movimiento estará afectado por la geometría del punzón y las condiciones locales de fricción. Los

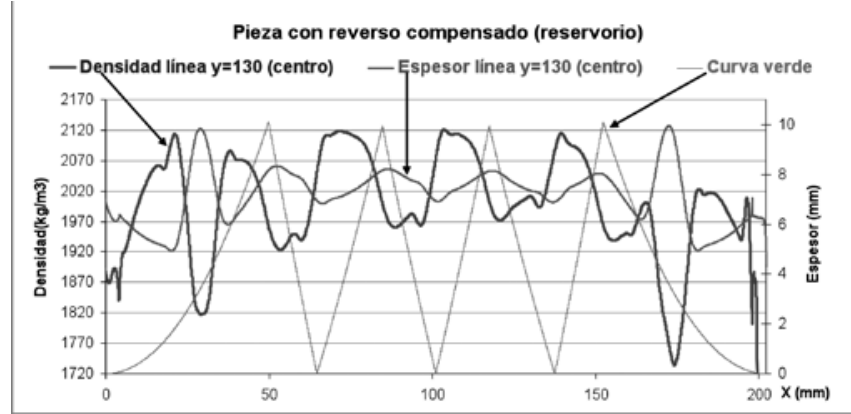

Figura 6. Da y espesor en una pieza con reverso compensado (reservorio)

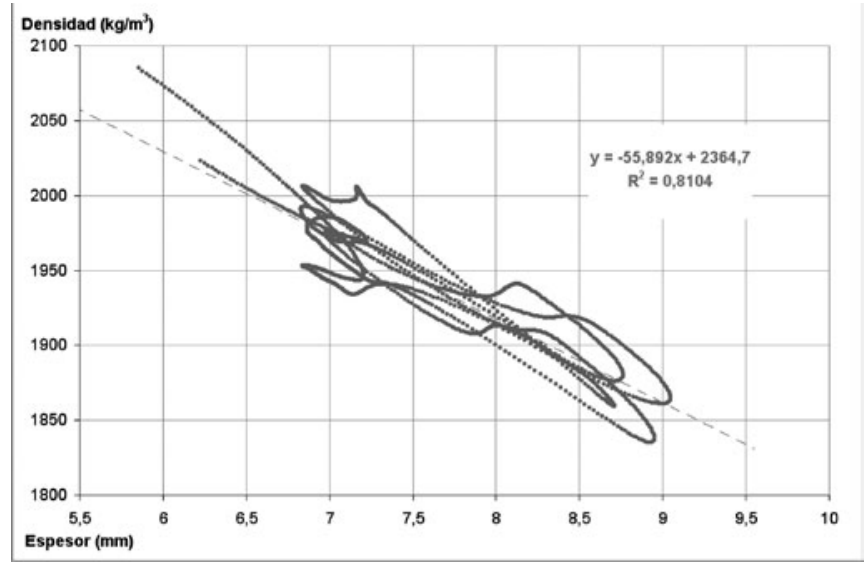

Figura 7. Da vs espesor para reverso con forma (reservorio) (todos los puntos) línea 17

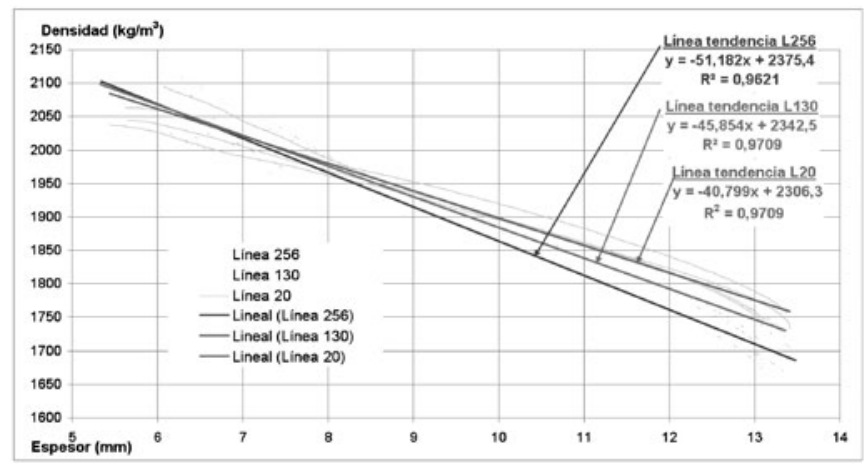

Figura 8. Da vs. espesor para reverso plano (sólo flancos de subida) 
resultados muestran que es posible optimizar la lubricación para minimizar el gradiente de Da en la tableta, evitando áreas de baja densidad ...".

En el caso estudiado, la fricción sola no explica los resultados, pues el punzón superior comienza a contactar con el polvo en la zona de la cresta y esta zona es la de menor Da (el mayor recorrido del punzón en el polvo no parece afectar). Por tanto, como se ha dicho en 2)-b) del punto 2.1, el arrastre mecánico por la "cuña" supera al efecto de la fricción polvopunzón. Hay que considerar la pendiente de la curva como un factor adicional. En cambio, la fricción sí explica la relación entre la Da y el espesor, pues a mayor espesor, más disipación energía y, por tanto, menor compactación.

En (2) se amplía el estudio y se enumeran los principales factores que influyen en la distribución de la Da en el prensado de tabletas: "comportamiento básico del polvo durante la compactación; la fricción entre el polvo y la pared del molde; la geometría del molde y punzones; la secuencia de movimiento del punzón; condiciones iniciales, que tienen que ver con el estado del polvo en el molde después de llenado". A la hora de fijar la Da objetivo, en la industria farmacéutica se ha establecido una regla: "la máxima heterogeneidad se alcanza cuando la relación entre la Da objetivo media y la densidad máxima del polvo está alrededor de 0,8".

En (4) se estudia la distribución de Da y el movimiento del polvo (celulosa microcristalina) en el prensado a 97,2 $\mathrm{MPa}$ de tabletas cilíndricas, tanto con caras planas como convexas, usando la Explicación de Train (5) para el desarrollo de los patrones de tensión/densidad, permitiéndole representar la evolución del patrón de presión en los compactos. Concluye que "la fricción es el principal factor que explica la distribución de $\mathrm{Da}$, de tal forma que reduciéndola se puede conseguir una distribución más uniforme; si se aumenta la presión así lo hace el rango de $\mathrm{Da}$, tanto para planas como curvas, pero más para las curvas, pues los efectos de la fricción son mayores; en el caso de planas, las regiones de alta densidad se dan en las esquinas superiores y en el corazón, con regiones de baja densidad en el centro de la parte superior y en las esquinas inferiores; en las curvas la región de alta densidad se da en el lateral de la tableta, donde el polvo está en contacto con el molde y el efecto de la fricción es superior". No obstante, cuando la relación altura/ anchura es baja, la importancia de la fricción se reduce.

Además en (6) se estudia en profundidad el efecto del movimiento lateral del polvo desde los extremos hacia el centro, pues juega un importante papel en la distribución de densidades (si éste no se diese, la densidad en los extremos en contacto con el punzón móvil sería mucho mayor de la observada). Para su medición se usa un arreglo de bolas metálicas en lugar de capas coloreadas. Concluye que el movimiento radial es mayor en, al menos, un factor de 3 para las tabletas curvas frente a las planas.

En la industria cerámica también se pueden encontrar estudios sobre la $\Delta \mathrm{Da}$, pero sólo para punzones planos, (7) relacionándola con la fricción entre la pasta y la pared del molde, pudiendo atribuir las regiones de alta Da al relativamente elevado desplazamiento del polvo cercano al punzón móvil junto a la pared del molde. En (8) se estudia el efecto del método de prensado y la geometría del molde, y concluye que para un molde cilíndrico y un único punzón móvil, el rango de Da es mayor que para el caso del prensado de doble efecto. Lo contrario ocurre para un molde en forma de bloque, que sería más parecido al caso aquí estudiado.
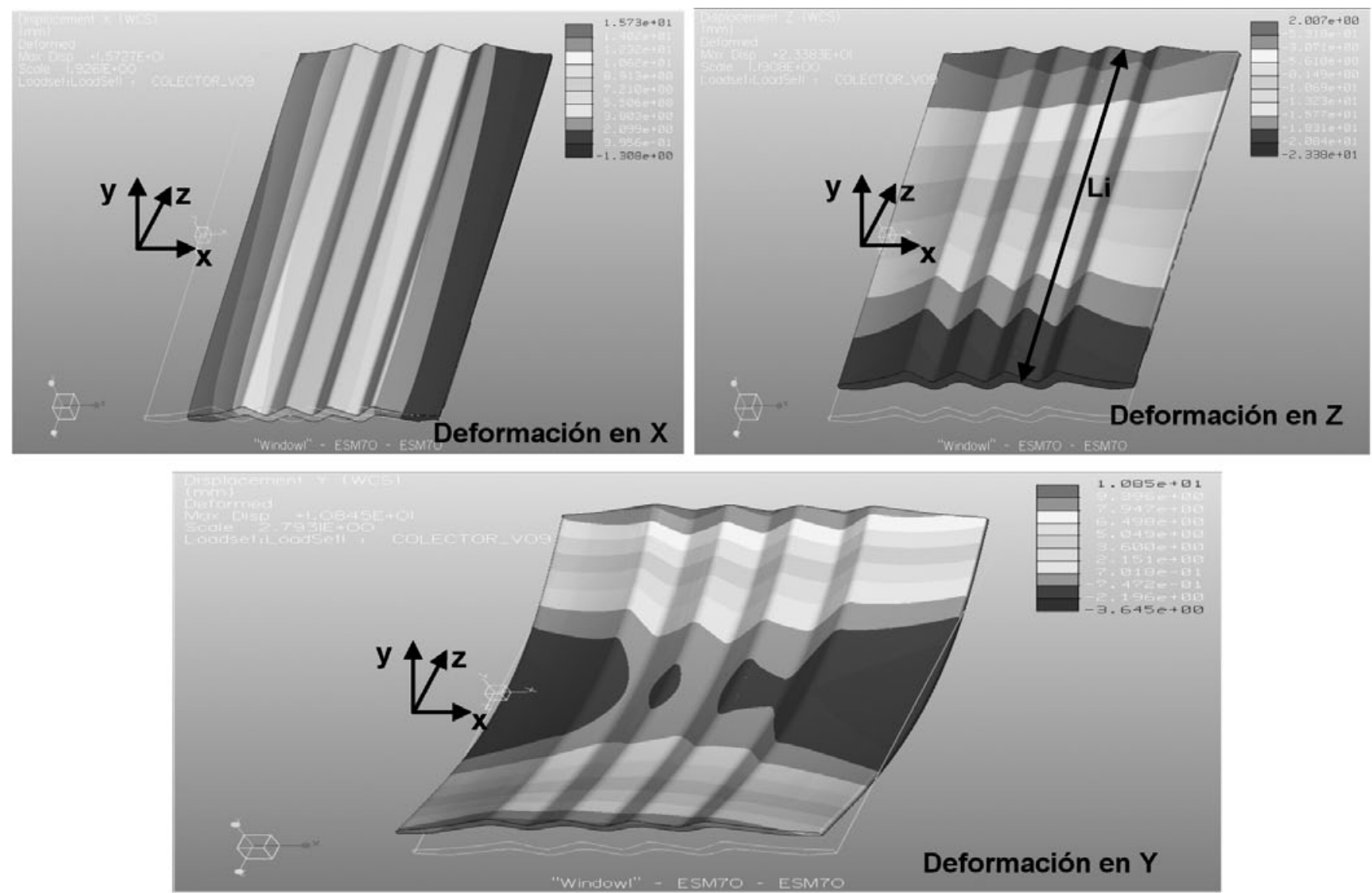

Figura 9. Valores de las deformaciones en piezas en cada eje obtenidas mediante simulación con MEF. 


\section{NUEVAS ESTRATEGIAS}

Más allá de la simple compensación analítica [en el plano XZ] del efecto cuadrático, por tratarse de perfiles curvos, de la contracción, típicamente anisótropa, en este trabajo se ha estudiado el efecto del perfil del punzón en la distribución de la Da que, como se ha dicho en el apartado 2, tiene un efecto directo e inevitable en la variación de la compactación (resultado de la $\Delta \mathrm{Pe}$ ), aunque con la ventaja de ser matemáticamente predecible y, por ello, compensable. En los experimentos llevados a cabo, se ha constatado otro fenómeno mucho más difícil de prever, como es el movimiento del polvo durante el prensado que genera $\Delta \mathrm{Da}$, básicamente originado por los dos mecanismos que se indican a continuación, si bien en la industria farmacéutica no se distingue entre ambos, aunque implícitamente (6) admite el arrastre por el punzón curvo:

- Arrastre mecánico, debido al efecto cuña del punzón, durante la primera fase del prensado (hasta que el punzón móvil contacta totalmente con el material).

- Difusión, por similitud con el transporte celular, debida al gradiente de presión efectiva, generado tanto en punzones planos como en curvos. Para éstos últimos se aumenta el efecto. Ésta difusión está limitada por la fricción, pues la fuerza del punzón es disipada y el polvo, alejándose de éste, menos compactado.

De esto se deduce la práctica imposibilidad de obtener una Da estrictamente uniforme en todo el cuerpo de la pieza, lo cual conlleva contracciones post-cocción diferentes, en mayor o menor medida según los valores de $\mathrm{Da}$, en diferentes puntos de la pieza. Este fenómeno, en piezas planas y en las ligeramente tridimensionales resulta en pequeñas deformaciones generalmente asumibles por los rangos de tolerancia habitualmente utilizados en el sector cerámico. Sin embargo, para el caso de piezas marcadamente tridimensionales en las que se exija, además, un elevado grado de precisión en la forma geométrica final, las variabilidades geométricas finales pueden ser claramente inasumibles.

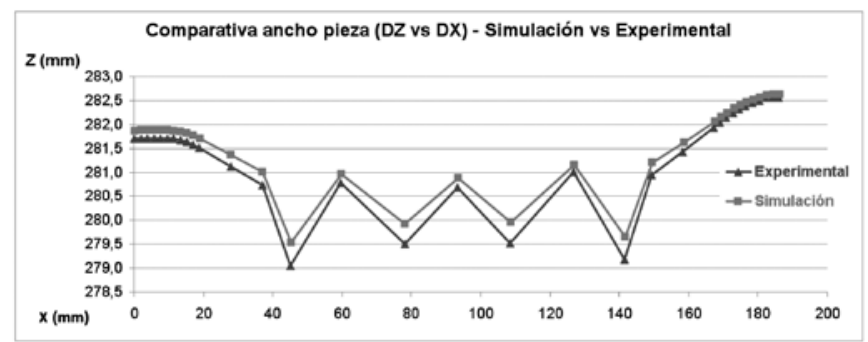

Figura 10. Dimensiones finales de piezas en ancho (valores $\mathrm{Li}$ en Figura 9) real/simulación

Una estrategia para tratar de minimizar este problema, derivado de variaciones difícilmente corregibles de la $\Delta \mathrm{Da}$, es ayudar a reducir las deformaciones geométricas vía compensación de la correspondiente geometría en verde. Con tal fin, se ha procedido ha realizar análisis de las contracciones de las piezas con una distribución heterogénea de la Da en su cuerpo, mediante el uso de simulación por el método de los elementos finitos (MEF), asignando coeficientes de contracción diferentes a cada zona de la pieza de acuerdo a la Da esperada y/o medida.

Los resultados de simulación han sido contrastados con los valores experimentales medidos con una MMC Brown \& Sharpe (DEA), modelo Mistral, observándose una correlación importante. Esta herramienta permite evaluar el nivel de afección en la geometría por parte de la diferencia de Da, de modo que se puede tener una predicción que permita compensar la geometría en el molde, así como evaluar el nivel máximo de heterogeneidad de la Da admisible y su distribución.

La industria farmacéutica, junto a la de la pulvimetalurgia, hace uso de las técnicas MEF para estudiar las diferencias de densidad que se producen durante el proceso de prensado de los polvos y el subsiguiente efecto de la liberación de tensióndeformación almacenada. Les interesa estudiar, al menor coste y mayor rapidez, el efecto de una geometría dada sobre la tasa de errores.
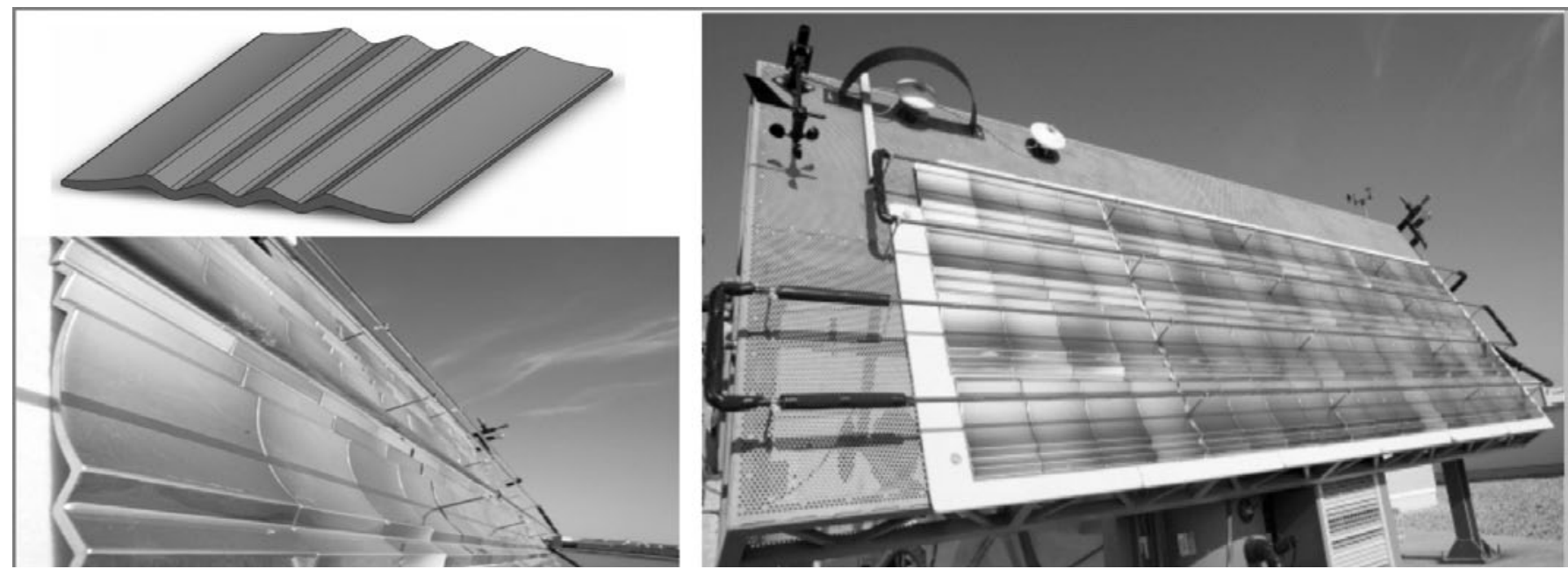

Figura 11. Ejemplo de aplicación de piezas cerámicas curvas con especificaciones geométricas de elevada precisión obtenidas mediante prensado con métodos de la cerámica plana, desarrollado en el proyecto CCPC "Colector de concentración cilindro-parabólico de material cerámico para energía solar térmica" financiado por el CDTI 


\section{CONCLUSIONES}

La metodología descrita permite aplicar al diseño de moldes y punzones técnicas para compensar las deformaciones. Éstas pueden usarse en casos de contracción anisótropa y geometrías no lineales (cuadráticas, splines, etc.), previendo las compensaciones necesarias en la geometría en verde para lograr la esperada, abriendo un amplio campo de aplicación en ingeniería y arquitectura.

Es factible obtener por prensado uniaxial piezas con un marcado perfil curvo, respetando un límite a las pendientes máximas.

La estrategia usada reduce el rango de $\Delta \mathrm{Da}$ introducida por el movimiento de la pasta y la variación de espesor. La mera eliminación del movimiento mecánico de la pasta, como ocurre en el caso del reverso plano, no es suficiente. La modificación de la geometría del reverso para habilitar un reservorio es prometedora, pero se necesita un procedimiento de evaluación de resultados menos costoso que la prueba y error, como puede ser la simulación por ordenador. Queda pendiente solucionar la $\Delta \mathrm{Da}$ introducida por la geometría.

Tratar de conseguir $\Delta \mathrm{Da}$ muy pequeñas en este tipo de piezas, de modo que se minimicen las deformaciones diferenciales, resulta muy complicado. Cuando se pretenden obtener geometrías cocidas complejas con un elevado nivel de precisión, este aspecto es muy relevante. Los análisis de simulación mediante MEF teniendo en cuenta las Da en cada zona de la pieza, han dado resultados muy satisfactorios en cuanto a la predicción de la geometría final de una pieza cocida y, por tanto, de las deformaciones diferenciales debidas a la heterogeneidad de la Da. Esto permite abrir un campo de trabajo de modo que mediante estas técnicas se puedan calcular y establecer compensaciones a aplicar a la geometría en verde de modo que una vez cocida la geometría final sea la esperada, o bien, establecer cuáles podrían ser las variaciones máximas de Da y su distribución en una pieza de modo que las deformaciones residuales resultantes en la geometría final estuviesen dentro de lo que funcionalmente se considerase acepta Una posible aplicación de lo expuesto en este trabajo, y que ha sido el origen del mismo, es la obtención de geometrías curvas de elevada precisión para el desarrollado de un colector solar térmico cilíndrico-parabólico de concentración realizado en material cerámico para integración en edificios, en el que se requiere que las diferentes facetas parabólicas (tanto de cada pieza como de las diferentes piezas) presente el mismo foco con errores de escasísimos milímetros con el fin de conseguir la eficiencia requerida. Los resultados obtenidos han sido muy satisfactorios.

\section{BIBLIOGRAFÍA}

1. ITC. Densexplorer. Noticias. [En línea] 26 de 10 de 2009. http://www. itc.uji.es / notDestacada / Paginas / ElITCradiografialasbaldosaspara asegurarcalidadyseguridadeneltrabajo.aspx.

2. Sinka I. C., Burch S. F., TweedJ. H., Cunningham J. C., Measurement of density variations in tablets using X-ray computed tomography, International Journal of Pharmaceutics (Elsevier), V. 271, 215-224 (2004).

3. Sinka I. C. , CunninghamJ. C. , Zavaliangos A. The effect of wall friction in the compaction of pharmaceutical tablets with curved faces: a validation study of the Drucker-Prager Cap model, Powder Technology (Elsevier), V. 133, 33-43 (2003).

4. Eiliazadeh B., Briscoe B. J., Sheng Y., Pitt K., Investigating Density Distributions for Tablets of Different Geometry During the Compaction of Pharmaceuticals, Particulate Science and Technology (Taylor \& Francis Inc), V. 21, págs. 303-316. (2003).

5. Train D., Transmission of forces through a powder mass during the process of pelleting, Trans. Inst. Chem. Eng., V. 35, 258-266 (1957).

6. Eiliazadeh B., Pitt K., Briscoe B. J., Effects of punch geometry on powder movement during pharmaceutical tabletting processes, International Journal of Solids and Structures (Elsevier), V. 41, 5967-5977 (2004).

7. Carlone P., Palazzo G., Computational modeling of the cold compaction of ceramic powders, International Applied Mechanics (Springer New York), V. 42 (10), 1195-1201 (2006).

8. Carlone P., Palazzo G. Cold compaction of ceramic powder: Computational analysis of the effect of pressing method and die shape, International Applied Mechanics (Springer New York), V. 43 (10), 1174-1178 (2007).

9. Cante J. C., Riera M. D. , Oliver J., Prado J. M. , Isturiz A., Gonzalez, C. Flow regime analyses during the filling stage in powder metallurgy processes: experimental study and numerical modeling, Granular Matter (Springer Berlin/Heidelberg), V. 13 (1), 79-92 (2011).

Recibido: 01/03/2012

Aceptado: 11/04/2012 